\title{
Innovation and Practice of Cable-Pylon Anchorage Zone Using Group Aggregated Anchor System
}

\author{
Yonggao Yin ${ }^{1, a}$, Qian Li ${ }^{2, a,}$, , Jiangguo Lv ${ }^{1, b}$, Zhu Yu ${ }^{1, c}$ \\ ${ }^{1}$ Anhui Transportation Holding Group Co., Ltd, Hefei, China \\ ${ }^{2}$ Research Institute of Highway Ministry of Transport, Beijing, China \\ a601060901@qq.com,b12771320@qq.com,c 348361184@qq.com
}

\begin{abstract}
Keywords: Cable-Stayed Bridge, Cable-Pylon Anchorage Zone, Group Aggregated Anchor System, Structure, Construction
\end{abstract}

\begin{abstract}
With the development of the cable-stayed bridge, the anchorage form on pylon of cable-stayed has been improved and innovated continuously, and the anchorage methods such as circumferential prestressed anchorage, steel anchor beam and steel anchor box have been gradually formed and developed, which further increases the span of cable-stayed bridge and meets the social needs of economic development and environmental integration. The group aggregated anchorage system between cable and pylon is a kind of anchorage form outside the pylon, which has the characteristics of clear force transmission, simple structure and high construction efficiency. It has been successfully applied in Chizhou Yangtze River Bridge for the first time. The main span of Chizhou Yangtze River Bridge is $828 \mathrm{~m}$, and the cable-stayed bridge with spatial cable plane of two towers is constructed. Six steel beams are deployed between tower legs to anchor 54 pairs of cables respectively. The steel beams and the concrete tower columns are effectively connected by prestressed anchors, shear nails and short steel bars, which could transfer the cable force to the tower column reliably. This kind of anchoring system has clear force transmission, which could reduce the tensile stress of concrete tower column and the risk of concrete cracking. Meanwhile, the steel beam could be constructed by the engineering manufacture and the field installation, which could reduce the working time at height, further the construction quality and safety could be controlled. Based on the construction of Chizhou Yangtze River Bridge, this paper mainly introduces the proposal, construction, key construction technology and engineering application effect of group aggregated anchorage system. The engineering practice proves that this new type of anchorage could not only meet the basic requirements of the intrinsic safety of the bridge, but integrate with the regional culture to create the beauty of natural harmony as well.
\end{abstract}

\section{Introduction}

The construction of cable-stayed bridge in China is relatively late. The first cable-stayed bridge is Yunyang Bridge in Sichuan Province, which was built in 1975. The span of this double tower cable-stayed bridge is $75.84 \mathrm{~m}$. The construction of this bridge has promoted the development of cable-stayed bridge in China. In the following ten years, the dense cable system was developed and applied. The completion of Shanghai Maogang Bridge and Jinan Yellow River Bridge brought the construction of cable-stayed bridge into the 200-meter era. Since the 1990s, due to the needs for crossing wider river, cable-stayed bridge has been developed rapidly, and the main girder has been lighter ${ }^{[1]}$. At this stage, the span of cable-stayed bridge has exceeded $600 \mathrm{~m}$, even reached the kilometer level. A series of landmark bridges have been built in succession, including Shanghai Yangpu Bridge, Wuhan Baishazhou Bridge, Sutong Yangtze River Bridge, Hong Kong 
Stonecutters Bridge. The breakthrough and innovation of cable-stayed bridge construction technology has made the development and construction of cable-stayed bridges in China into the world's advanced ranks.

The span capacity of cable-stayed bridge is improved continuoustly, which means that the load level would increase, and the structural safety problem would become more prominent. The anchorage structure of stay cable and bridge tower is an important structure to transfer the local concentrated force of a cable to the tower column safely and uniformly ${ }^{[2]}$. The structure of Cable Tower Anchorage Zone is closely related to many factors, such as the layout and quantity of cables, the form and structure of cable tower. Besides, the stress state is complex, which is one of the focuses and difficulties in the design and construction of cable-stayed bridge ${ }^{[3-4]}$. The common forms of cable anchorage include side wall anchorage, steel beam anchorage, cross anchorage, steel anchor box, etc. ${ }^{[5]}$. With the development of cable-stayed bridge, the cable tower anchorage structure is also constantly improved and innovated.

\section{Anchorage of Cable and Concrete Tower}

In the early stage, the tower column in the anchorage section of medium and small span cable-stayed bridge was solid section, and the cross anchorage structure was mostly used, while it is rarely used at present. In recent years, non-staggered anchorage structure has been widely used in long-span cable-stayed bridges, such as circumferential prestressed anchorage, steel anchor beam and steel anchor box anchorage.

The circumferential prestressed anchorage method uses the external force generated by the prestressed steel bundle to balance the internal force generated by the cable force in the tower column. It has the characteristics of simple structure, low engineering cost and small maintenance workload in the later stage. It is widely used in Yangpu Bridge, Jingzhou Yangtze River Highway bridge, South Branch of the Second Nanjing Yangtze River Bridge, etc. However, due to the extrusion of prestressed tendons in the bending zone, the stress distribution is complex, and the values of elongation and friction coefficient are not clear, and the data measured by the test are quite discrete ${ }^{[6-7]}$. In addition, according to the related research results ${ }^{[8-9]}$, when the span exceeds $800 \mathrm{~m}$, the prestressed tendon tonnage is very large, and the thickness of tower column wall and the number of prestressed steel strands will increase, resulting in prestressed tension. Therefore, the circumferential prestressed anchorage is not suitable for the super long-span inclined bridge. Instead, the steel anchor beam steel (or concrete) corbel combination anchorage form and steel anchor box anchorage form are used.

The steel beam in the anchoring form of steel beam is installed inside the hollow tower column and supported on the concrete or steel corbel of the inner wall of the concrete tower column, which is an independent and stable component, and mainly bears the horizontal tension, vertical component force and eccentric bending moment of the cable. The steel beam sustains the horizontal component of the stay cable, which could effectively reduce the tension stress produced by the horizontal component of the cable in the concrete tower column. This kind of anchorage structure is simple and clear, and has been widely used in cable-stayed bridges at home and abroad, such as Jintang Bridge, Jingyue Yangtze River Highway Bridge, North Branch of Xiazhang Bridge, etc. After continuous improvement, the steel beam-steel corbel anchorage form could adapt to the space cable surface, easy to achieve the industrial production of integral assembly, and the construction could be faster.

Compared with prestressed anchorage and steel anchor beam anchorage, the steel anchor box anchorage is widely used in cable-stayed bridges with span greater than $600 \mathrm{~m}$, such as Sutong Yangtze River Highway Bridge, Stonecutters Bridge, Normandy Bridge in France, Hangzhou Bay 
Sea Crossing Bridge, etc. The main components of steel anchor box are composed of pulling plate along bridge direction, end bearing plate, web, bearing plate under anchor, anchor plate, stiffening rib, working platform and others ${ }^{[10]}$. The horizontal component of the stay cable is borne by the steel anchor box pulling plate, and the vertical component of the stay cable is transmitted to the concrete tower through the stud. The advantages of steel anchor box anchorage are similar to that of steel anchor beam, which could be made in factory, installed on site, and easy to detect and maintain. Moreover, the steel structure has reliable stress and easy to control the position and angle of anchor point. However, due to the large amount of steel used, the internal space of the tower column is required to be large, and the hoisting equipment and installation demand high accuracy. What's more, under the condition of the cable balanced horizontal force, the concrete of the tower column would also produce tension stress, which is unfavorable to the durability of the structure.

According to statistics, the steel-concrete composite anchorage system is used for built cable-stayed bridges where the span is more than $600 \mathrm{~m}$. This anchorage system could full perform the tensile strength of steel and the compressive properties of concrete materials, and could realize factory prefabrication, controllable construction quality, high safety and easy maintenance, which has been widely used in super long-span cable-stayed bridges.

\section{The Group Aggregated Anchorage System and Construction Technology}

The Group Aggregated Anchorage System

The group aggregated anchorage system is a relatively new type of stay cable tower anchorage system. The cable is grouped and separately anchored on multi-channel steel beams between towers. The steel beam and concrete tower column are reliably connected by prestressed anchor rod, shear nail and short steel bar, so as to achieve the external anchorage of stay cable tower end $^{[11,12]}$. The cable plane layout of the structure of the grouped aggregated cable tower is more novel and unique, which enhances the visual appreciation and impact force. It has been proposed and applied in Chizhou Yangtze River Bridge for the first time.

Chizhou Yangtze River Highway Bridge (see Figure 1) is a cable-stayed bridge with two pylons and spatial cable plane with main span of $828 \mathrm{~m}$. As is known to all, every bridge across the river is a landmark structure of a city, which needs functional, cultural and aesthetic value. In the design process of the main tower of the bridge, since Chizhou Yangtze River Highway Bridge is located in Chizhou Jiuhua Mountain Buddhist holy land, the construction team creatively integrates Buddhist cultural elements into the main tower design of the bridge. The main tower adopts the vase type concrete cable tower scheme. In the upper tower section, 54 pairs of stay cables divided into 6 groups and anchored on the steel cross beams between towers, so as to create the radiation effect of Buddha beads beam, and closely echo the bridge aesthetics and regional culture.

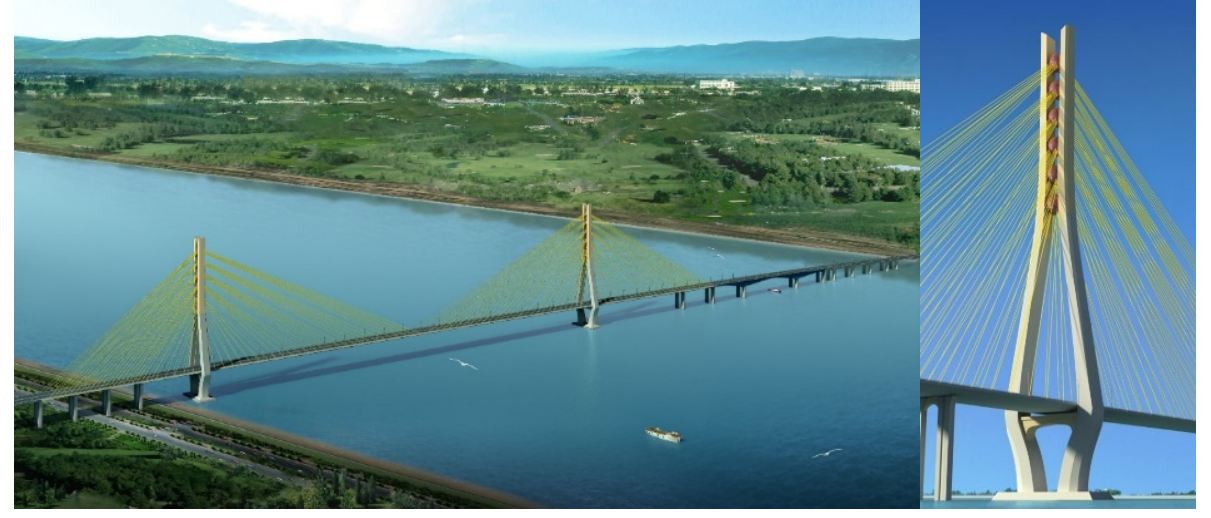

Fig. 1 Effect Drawing of Chizhou Yangtze River Highway Bridge 
The steel cross beam between towers of the bridge adopts box structure. There are three diaphragms set in the middle along the transverse direction of the bridge, and the anchor box of the cable is set between the diaphragms (see Figure 2). The end of the steel beam is welded with the embedded steel plate on the concrete tower wall, and the high-strength anchor rod is used to connect the steel beam and the tower column. One end of the anchor bolt is anchored on the anchorage bracket of the steel beam side, and the other side is anchored on the steel anchor plate in the concrete tower column. The tension is carried out by the step-by-step tensioning and anchoring technology. Whar's more, the embedded steel plate is welded with the vertical reinforcement of the outermost layer of the tower column through short steel bars. The bridge load is transmitted to the tower column through the cable, and the horizontal component of the cable is borne by the vertical and horizontal steel plate of the steel beam, meanwhile the vertical component is transferred to the steel beam, which produces bending moment and shear force at the beam end of the steel beam. The bending moment is mainly borne by the prestressed anchor rod, and the steel-concrete joint surface produces large static friction force by using the split anchor rod, which could resist the shear effect. Such structure could significantly improve the force of concrete tower columns.

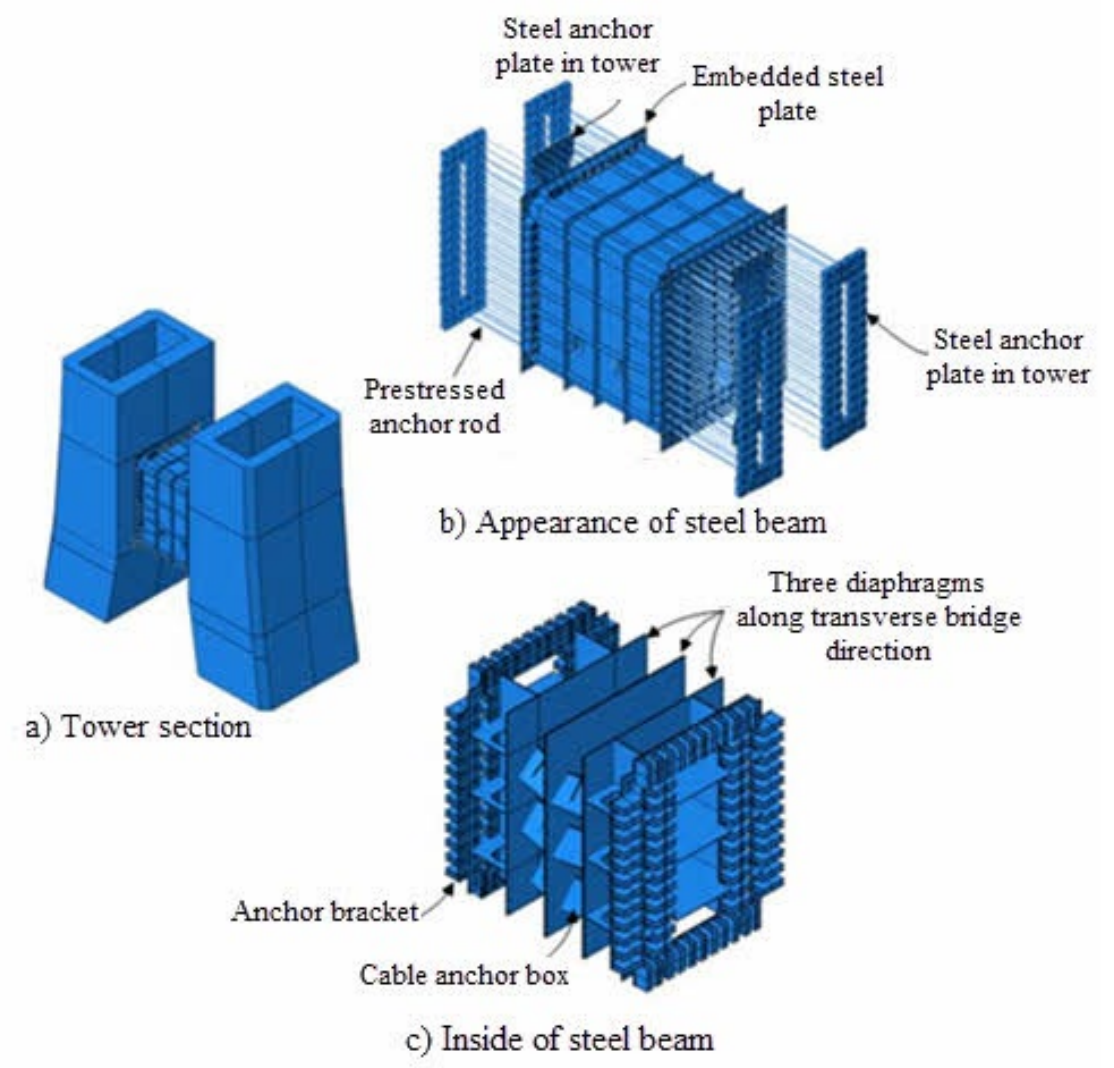

Fig. 2 Structural Diagram of Steel Beam between Towers

Key Construction Technology of the Group Aggregated Anchorage System

The construction of the group aggregated anchorage system mainly involves the fabrication and processing of the steel beam between the towers, the installation of the steel beam between the towers, and the installation and tension of the stay cables. The first three items are closely related to the anchoring structure and are the important factors affecting the construction quality and safety of the tower columns. 
The steel beam between the towers of Chizhou Yangtze River Highway Bridge is $7.0 \mathrm{~m}$ high, $5.5 \mathrm{~m}$ wide along the bridge direction and $7.0 \mathrm{~m}$ wide in the transverse direction. The steel beam is divided into three sections along the transverse direction of the bridge, and the middle block is divided into two blocks along the longitudinal direction of the bridge. The four blocks are connected by high-strength bolts. The maximum weight of a single block is $68 \mathrm{t}$ (see Figure 3 ). Since there are many groups of cable anchor boxes and diaphragms inside the steel beam, many small boxes and lattice space are formed inside the steel beam. The welding seam is dense, and the plate is thick; furthermore, there are many penetration welds and deep groove welds. The welding deformation control and weld quality would directly affect the site construction accuracy and structural bearing performance.

Welding deformation could be ensured by assembly process, welding method and welding sequence. The middle block could be assembled according to the sequence of separately assembling anchor box unit, middle web, side web, top and bottom plate, joint plate, reinforcement plate and stiffening rib, in which the key point is the angle accuracy of anchor head. Besides, the assembly sequence of side block could be organized as follows: embedded steel plate positioning, web, wall plate, top and bottom plate, stiffening plate, anchor plate, stiffening rib and drilling connection hole, in which the key point is to control the perpendicularity of main frame structure and embedded plate.
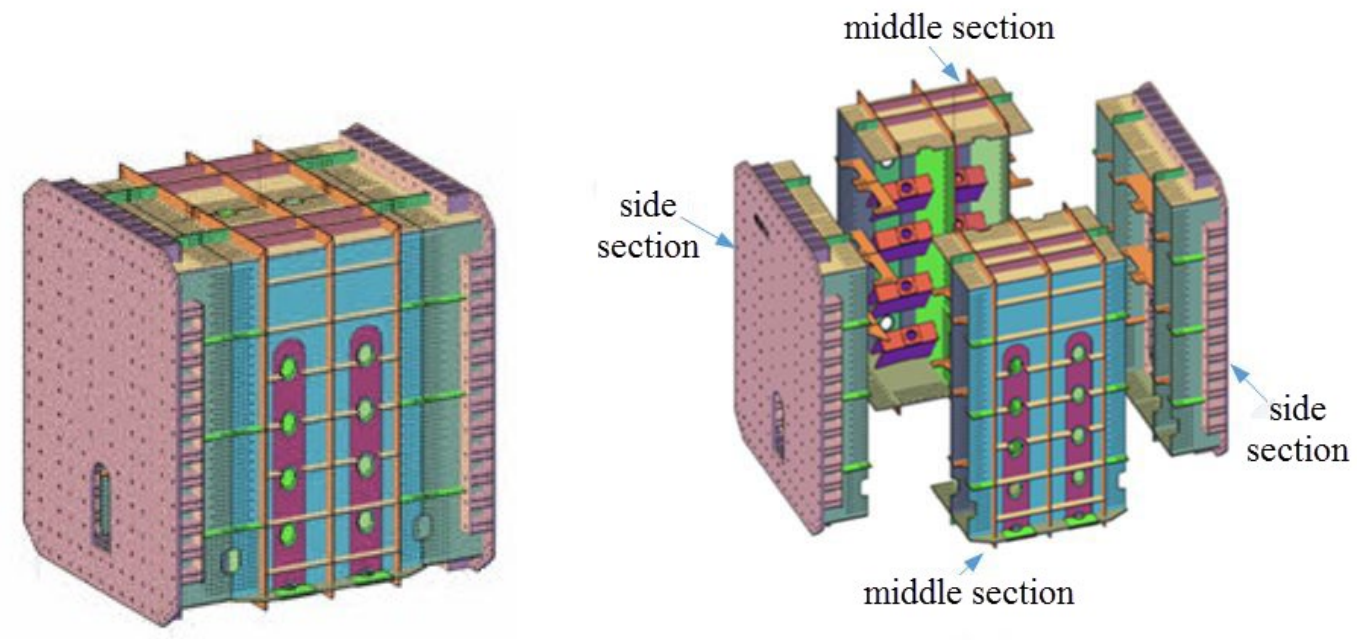

Fig. 3 Diagram of Blocks of Steel Beam between Towers

According to the characteristics of the steel beam, combined with the mature construction method of the hoisting operation of high tower and large components, the steel beam installation scheme of "large tonnage tower crane + section steel bracket + three-way Jack positioning system + shape steel and steel wire rope soft hard combination anti overturning system" is proposed (see Figure 4). The installation process is designed as follows: embedding steel beam installation bracket, installing steel beam mounting bracket, large tonnage tower crane hoisting steel beam according to the sequence of side section, middle section and side section, using three-way positioning jack to adjust position, tightening splicing plate and high bolt between steel beam segments, installing steel beam high-strength anchor rod, casting tower column concrete for twice, and tensioning high-strength anchor rod. 


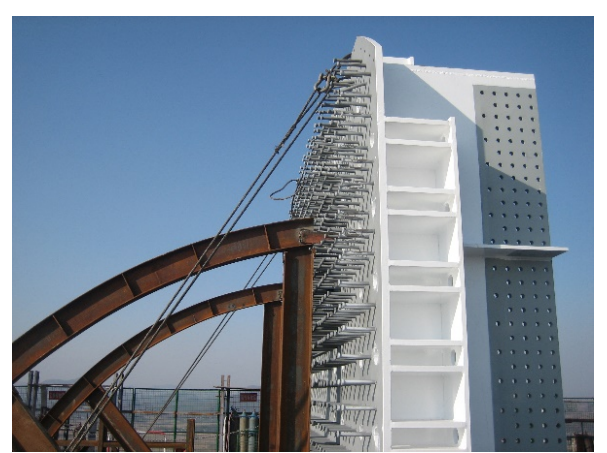

Fig. 4 Wind Rope Pulling and Section Steel Support Locking

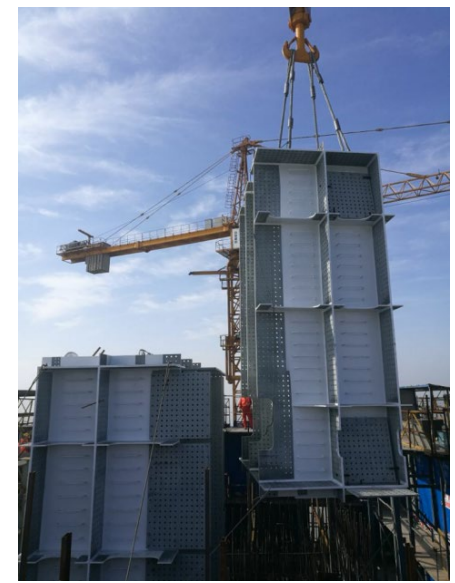

Fig. 5 Installation of Middle Section of Steel Beam

\section{Effect of Engineering Application}

Taking the Chizhou Yangtze River Highway Bridge project as the research object, and focusing on the design and construction of the group aggregated anchorage system, a series of theoretical analyses, numerical simulations, model tests and process tests are performed. From theoretical analysis to experimental research and real bridge detection, and then to engineering practice, the technological achievements, including the design and construction key technology of the grouped aggregated anchorage system, have been continuously supplemented and improved. According to the scientific research and engineering practice, the grouped aggregated anchorage system has the characteristics:

(1) The external anchorage of the stay cable tower would not occupy the internal space of the tower column, and the structural size of the main tower is optimized.

(2) The vertical component of the cable force is transformed into the bending moment at the end of the beam, which is borne by the high-strength anchor rod, and would not act on the tower wall directly, so as to reduce the concrete tensile stress and avoid the concrete tower column cracking.

(3) The construction of steel beam outside the tower could greatly improve the installation efficiency. According to calculations, compared with the independent anchorage system, the installation efficiency of single group of aggregated anchorage system could be increased by about $50 \%$.

(4) Using small steel beam instead of concrete beam, there is no need to set up large support platform on site. Steel beams could be prefabricated in factory and assembled on site, which could improve accuracy, save materials, shorten construction period and reduce safety risk. 
(5) After the installation of the first steel beam of the cable tower is completed, the cable tower has a stable structure, and the upper structure of the cable-stayed bridge could be normally promoted according to the construction steps of the tower and beam, and the synchronous construction of the tower and beam could be reached. Moreover, one steel beam could meet the hanging of multiple pairs of stay cables, which is easy to install and would greatly save the construction period.

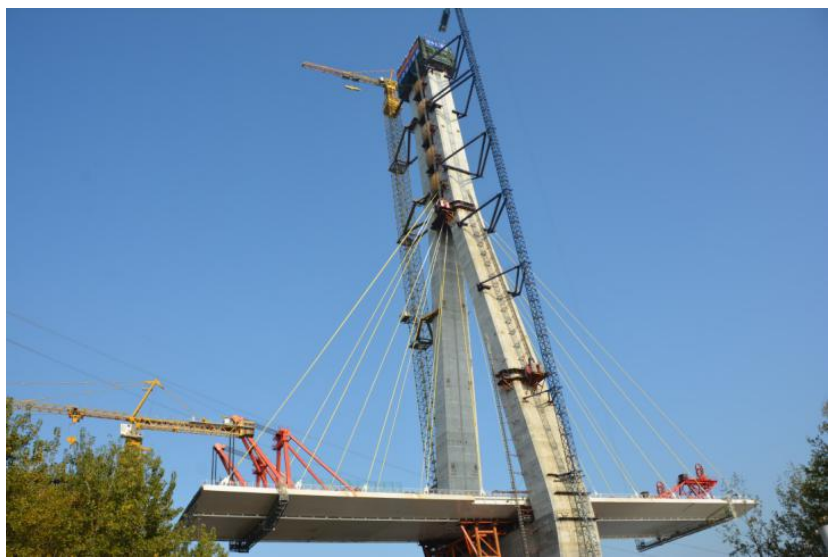

Fig. 6 Synchronous Construction Site of Tower and Beam

\section{Summary}

Based on the development trend of cable-stayed bridge construction and the demand of long-span construction, the group aggregated anchorage system could make full use of the material characteristics. Based on that, the structure is simple, the force transmission being clear, and the efficiency is high. This system could further improve the durability of the structure, as well as the span capacity and bearing capacity of the cable-stayed bridge. Stresses of three diaphragms and cable anchor box inside steel beam should be monitored in construction and operated stage. And the prestressed force of anchor rod need to be tested at regular intervals to ensure that steel beam can be connect tightly with concrete tower.

\section{References}

[1] Zhuanghong Lou. Collection of papers on bridges by Zhuanghong Lou [M]. Beijing: China Communications Press, 2004.

[2] Shilin Liu, Zhitao Liang, Jinlong Hou, Fanchao Meng. Cable-stayed Bridge [M]. Beijing: China Communications Press, 2002.

[3] Changpeng Liu, Xigang Zhang, Rengui Wang, Feng Wen. Study on Stress and Structural Characteristics of Cable Tower Anchorage Zone of Composite Structure [J]. Highway, 2012 (1): 117-121.

[4] Qifen Wei, Ying Chang, Wenhai Ye, Kai Bai. Structural Design and Calculation of Cable Tower Anchorage Zone of Edong Yangtze River Highway Bridge [C]. The 20th National bridge Academic Conference, Wu Han, Hu Bei Province, May 15 ${ }^{\text {th }}, 2012$.

[5] Industry recommended standards of the People's Republic of China. Guidelines for Design of Highway Cable—stayed Bridge [S]. Beijing: China Communications Press, 2007.

[6] Lu Shen, Wenliang Qiu. Analysis and Experimental Study on Prestress Loss of Circumferential Steel Tendons in Anchorage Zone of Pylon of Long Span Cable-stayed Bridge [J]. Journal of Water Resources and Architectural Engineering, 2016, 14(2): 97-99. 
[7] Yiqiang Xiang, Shaoping Yi, Xiaoqing Du, Xing Xu. Study on Full Scale Model of Cable-stayed Pylon Segment of Nancha Bridge of The Second Nanjing Yangtze River Bridge [J]. China Civil Engineering Journal, 2000, 33(1): 16-22.

[8] Byung-Wan Jo, Yunn-Ju Byun, Ghi-Ho Tae. Structural Behavior of Cable Anchorage Zones in Prestressed Concrete Cable-stayed Bridge[J]. Canadian Journal of Civil Engineering, 2002, 29(1): 171-180. https://doi.org/10.1139/101-087

[9] Burdet O. Analysis and Design of Anchorage Zones in Post-Tensioned Concrete Bridges[D]. Austin: University of Texasat Austin, 1990.

[10] Xigang Zhang, Yuqing Liu. Anchorage structure of composite cable tower [M]. Beijing: China Communications Press, 2010.

[11] Canwen Yang, Qiang Zhang, Jianhua Shi. Design of Main Navigable Span Bridge of Chizhou Yangtze River Highway Bridge [J]. Bridge Construction, 2016, 46(4): 92-96.

[12] Po Wen, Qiang Zhang, Jianhua Shi. Design of Anchorage Structure for Upper Beam of Main Bridge Tower of Chizhou Yangtze River Highway Bridge [J]. Bridge Construction, 2017, 47(2): 83-88. 